\title{
Uma metanálise de dissertações e teses brasileiras que utilizaram a teoria do Pensamento Matemático Avançado
}

\author{
A meta-analysis of brazilian dissertations and theses that used the theory of \\ Advanced Mathematical Thinking
}

Paulo Ferreira do Carmo

Sonia Barbosa Camargo Igliori

\begin{abstract}
Resumo: Após um certo período de publicações sobre um determinado tema, pesquisadores sentem a necessidade de analisá-las para verificar tendências dessas publicações. 0 objetivo deste artigo é apresentar uma metanálise de pesquisas brasileiras baseada em alguns aspectos dessas produções que utilizaram noções de Pensamento Matemático Avançado em seus referenciais teóricos. Para isso, foram utilizadas 21 dissertações e 5 teses destacando-se aspectos considerados importantes dessas pesquisas. Esta investigação é parte de uma pesquisa de doutorado, que visa analisar as diversas concepções do PMA em publicações brasileiras. A metodologia utilizada nas análises segue as orientações da análise de conteúdo. Constatou-se que as pesquisas se concentram na região sudeste e no Ensino Superior, e que a maioria destas pesquisas foi de caráter empírico, mostrando a potencialidade desta teoria cognitivista para 0 ensino e aprendizagem de conceitos matemáticos.
\end{abstract}

Palavras-chave: Pensamento Matemático Avançado. Metanálise. Teoria Cognitivista. Dissertações e Teses Brasileiras. Educação Matemática.

Paulo Ferreira do Carmo

Doutor em Educação Matemática. Professor da Universidade Federal de Mato Grosso (UFMT), campus Araguaia. Mato Grosso Brasil.

iD orcid.org/0000-0001-7029-6335 $\bowtie$ paul02012carmo@gmail.com

Sonia Barbosa Camargo Igliori Doutora em Matemática. Professora do Programa de Estudos Pós-Graduados em Educação Matemática da Pontifícia Universidade Católica de São Paulo. São

Paulo, Brasil.

orcid.org/0000-0002-6354-3032

$\bowtie$ sigliori@pucsp.br

Recebido em 09/07/2020 Aceito em 07/09/2020 Publicado em 09/11/2020 and in higher education and that most of this research was empirical in character, showing the potential of this cognitive theory for the teaching and learning of mathematical concepts.

Keywords: Advanced Mathematical Thinking. Meta-analysis. Cognitive Theory. Brazilian Dissertations and Theses. Mathematics Education.

\section{Introdução}

A sociedade contemporânea exige, cada vez mais, dos indivíduos escolhas e tomada de decisões em diversas situações, para estruturar dados e informações, fazer previsões, avaliar 
riscos quantitativamente, relacionar os conhecimentos e aplicá-los em novas situações. Propiciar essas competências é um dos papéis do ensino da Matemática, quando se considera o conhecimento matemático, para além de favorecer o conhecimento de técnicas para efetuar operações. Conhecer a formação do pensamento matemático e seu papel nos conhecimentos matemáticos é essencial para o desenvolvimento do ensino e da aprendizagem da Matemática. É um estudo sobre essa temática que se apresenta neste artigo, com enfoque na formação do pensamento matemático.

O conhecimento matemático desempenha um papel importante na formação do pensamento humano; é ele que possibilita a formação de uma espécie de pensamento, em geral compreendido como formas de pensar que municiem a formação de competências como classificar, contar, ordenar, realizar operações aritméticas, algébricas, geométricas etc. Mas se sabe que há outras, como criar, desenvolver, fazer escolhas etc.

Alguns pesquisadores, abordados neste artigo, voltados para pesquisas cognitivistas, separam esse pensamento em dois tipos — o Pensamento Matemático Elementar, relacionado às condições de manipular e operar objetos matemáticos (descrever para definir), e o Pensamento Matemático Avançado, que possibilita definir objetos matemáticos por meio dos próprios conceitos matemáticos (convencer para provar).

As pesquisas analisadas neste artigo indicam que é a transição do pensamento matemático do elementar para o avançado que tem causado dificuldades para o ensino e a aprendizagem na passagem da Educação Básica ao Ensino Superior.

As pesquisas em Educação Matemática no Ensino Superior têm crescido significativamente nas últimas cinco décadas e se apoiam nos constructos do Pensamento Matemático Avançado em seus referenciais teóricos. Mas falta consenso entre os pesquisadores que trataram do tema na caracterização desse tipo de pensamento.

Este artigo apresenta uma metanálise das pesquisas brasileiras com vistas a identificar como os pesquisadores brasileiros se posicionam frente ao descenso conceitual. Para tal, considerou-se: objetivo, resultados, nível de ensino, tema/conteúdo matemático nas pesquisas que se orientaram especialmente pelas ideias de Tall (2002) e Dreyfus (2002). Para alcançarmos nosso objetivo, esse artigo foi estruturado da seguinte forma: primeiro apresentamos alguns preceitos da teoria do Pensamento Matemático Avançado que irá subsidiar nossas análises e conclusões; segundo, apresentamos os procedimentos metodológicos e a metodologia de 
pesquisa utilizada nas análises para construção dos corpus do estudo; e, terceiro, apresentamos o corpus do estudo com as principais características das pesquisas e uma análise baseada em quantidades; e, por último, tecemos nossas considerações finais.

\section{Pensamento Matemático Avançado}

Para subsidiar o entendimento do leitor apresentamos algumas características da teoria do Pensamento Matemático Avançado (PMA) a partir da concepção de dois autores.

Dreyfus (2002) defende que a compreensão na mente do estudante é estruturada em uma sequência de atividades que possibilita a interação entre processos mentais e seus componentes - representação, visualização, generalização, classificação, indução, análise, sintetização, abstração e formalização. A aprendizagem depende dessa estruturação que ocorre de maneira intrincada. É essa estruturação, entre os diferentes componentes, que é denominada por Dreyfus (2002) de PMA e para ele são as diferentes interações entre esses componentes que sinalizam como ocorre a compreensão na mente do estudante. Esse autor considera que se pode pensar nos conteúdos de Matemática avançada de uma forma elementar e a distinção entre os dois tipos de pensamento reside na complexidade e na forma como se lida com essa complexidade. Para ele, há pouca distinção entre pensamento matemático elementar e avançado - a Matemática avançada foca nas abstrações de definição e dedução. Esse pesquisador ainda defende que "dentre os processos envolvidos no desenvolvimento do pensamento matemático avançado, 0 mais importante é a abstração, pois se um estudante desenvolve a habilidade de, conscientemente, fazer abstrações a partir de situações matemáticas, ele alcançou um nível avançado do pensamento matemático" (DREYFUS, 2002, p. 34).

Esse pesquisador afirma que a abstração e a representação são processos complementares que possuem direções opostas. Pois, se por um lado, um conceito muitas vezes é abstraído de suas representações, por outro, as representações advêm de um conceito abstrato.

Tall (2002) concebe o PMA sendo aquele gerido nas estruturas cognitivas do indivíduo produzido por uma grande variedade de atividades matemáticas, destinadas ao desenvolvimento de novas ideias que fundamentam e ampliam o crescente sistema de teoremas conhecidos. Ele considera que a passagem do pensamento matemático elementar (PME) para o PMA se inicia com as percepções de e ações sobre objetos em um mundo exterior, construído por meio de dois desenvolvimentos paralelos: um do visual-espacial para o formal-dedutivo; e outro de sucessivas 
encapsulações do processo para a formação do conceito, envolvendo a manipulação simbólica. Esses dois desenvolvimentos inspiram o pensamento criativo baseado em objetos formalmente definidos e em provas sistemáticas. Esse pesquisador diz que muitas das atividades que dão indicações da formação de um PMA também são do PME, mas a possibilidade de compreender uma definição formal e de realizar dedução é um fator que os diferenciam.

\begin{abstract}
A passagem do pensamento matemático elementar para o pensamento matemático avançado envolve a transição: do descrever para o definir, do convencer para o provar de uma maneira lógica com base nas definições. [...] é a transição da coerência da matemática elementar para a consequência da matemática avançada, com base em entidades abstratas que 0 indivíduo precisa construir pelas deduções das definições formais (TALL, 2002, p. 20).
\end{abstract}

Essa citação de David Tall revela que ele associa o PMA, principalmente, a conteúdos matemáticos do Ensino Superior, e, como referido antes, neste artigo, Tommy Dreyfus associa a relação desse pensamento a conteúdos de qualquer nível de ensino.

No âmbito da constituição da teoria que resulta na caracterização do PME e PMA, dois conceitos são formulados por Tall e Vinner (1981). São eles: imagem conceitual (concept image) e definição conceitual (concept definition). 0 primeiro se constitui na mente do estudante a partir de algo não verbal que ele associa à denominação atribuída a um determinado conceito; a estrutura cognitiva total associada ao conceito inclui todas as imagens mentais, todas as propriedades e todos os processos que the estão associados. O segundo é uma definição apresentada pelo estudante para explicar o conceito de modo verbal. Essa definição pode, e ocorre com frequência, não coincidir com a definição formal proposta pelos matemáticos. Um exemplo interessante dessa definição dada por estudantes de Cálculo: "Derivada é a tangente à uma curva, gráfico de uma função" (MEYER, 2003, p. 123). Tall e Vinner (1981) indicam ainda que o conhecimento da definição formal de um conceito pelo estudante não garante a compreensão desse conceito, é necessário a constituição de imagens em sua mente, mais precisamente o que se passou a denominar imagem conceitual.

O termo proceito (procept) foi introduzido em Gray e Tall (1994) para indicar a "amálgama de componentes de um conteúdo matemático na formação do pensamento matemático, quais sejam processo e conceito/objeto" (p. 7). Com esse conceito, eles explicitam a dualidade dos objetos matemáticos, que se apresentam na forma de processo antes de se constituírem na forma de conceito. Para esses autores, a combinação unificada das duas formas, processo e conceito (procept $=$ process + concept $)$ de uma noção matemática aparece nas práticas de trabalho dos 
matemáticos profissionais e daqueles que são bem-sucedidos nessa área. Um símbolo possibilita evocar o processo ou o conceito, como por exemplo $2+3$ pode ser visto como o processo de adicionar dois números ou o conceito de adição.

Mais recentemente, David Tall começou a construir uma estrutura simples a partir da herança genética da criança recém-nascida que é amplo o suficiente para cobrir o espectro de desenvolvimento dos diferentes indivíduos à medida que crescem. Na raiz desta crescente sofisticação está o uso da linguagem para comprimir um fenômeno complexo em um conceito cujo significado pode ser pensável e refinado por uma experiência discutida e conectada a outros conceitos pensáveis ricos em esquemas cognitivos (TALL, 2004). Essa estrutura é baseada em três diferentes mundos, mas interligados de desenvolvimento do pensamento matemático, dois dos quais dominam a Matemática Elementar, e o terceiro se desenvolve na Matemática Avançada, são eles:

- Mundo conceitual-corporificado - o mundo da percepção e reflexão das propriedades de objetos;

- Mundo proceitual-simbólico — o mundo da ação e simbolização de objetos;

- Mundo axiomático-formal - o mundo das definições formais e prova; que inverte a sequência de construção de significado considerando que ela se dá a partir de definições constituídas de conceitos formais conhecidos e em um conjunto de definições de uma teoria.

Esses são os elementos teóricos utilizados neste artigo.

\section{Procedimentos metodológicos e metodologia de pesquisa}

A metanálise pressupõe uma coleta de pesquisas que tratam do tema a ser reanalisado. Assim, para realizá-la tomou-se como metodologia a pesquisa de caráter bibliográfico, com vistas a obter dados/informações de documentação escrita para a análise de suas questões. Isso porque entende-se a metanálise, de acordo com Fiorentini e Lorenzato (2012), como uma revisão sistemática de outras pesquisas, visando a realizar uma avaliação crítica e/ou produzir novos resultados ou sínteses a partir do confronto desses estudos, transcendendo aqueles anteriormente obtidos.

As análises, por sua vez, ocorreram em conformidade à análise de conteúdo proposta por 
Bardin (2011), quando se considerou "um conjunto de técnicas de análise das comunicações que utiliza procedimentos sistemáticos e objetivos de descrição do conteúdo das mensagens" (p. 44). Tivemos como intenção considerar que o "conteúdo é a inferência de conhecimentos relativos às condições de produção (ou, eventualmente, de recepção), inferência essa que recorre a indicadores (quantitativos ou não)" (BARDIN, 2011, p. 44). Nesse caminho foram considerados os três polos cronológicos propostos na análise de conteúdo: a pré-análise; a exploração do material; e 0 tratamento dos resultados, a inferência e a interpretação.

A seleção dos estudos que compuseram o corpus para a análise foi realizada por meio de busca no Banco de Teses e Dissertações da Coordenação de Aperfeiçoamento de Pessoal de Nível Superior (CAPES), com a palavra-chave: "pensamento matemático avançado". Dos 38 estudos encontrados, foram selecionados 26 , sendo 21 dissertações e 5 teses. Por meio da leitura dos resumos foi feita a seleção daqueles estudos que incluíram PMA e/ou os conceitos relacionados a esse processo, conforme descrevemos anteriormente. Esse último critério de buscar publicações mais recentes teve por objetivo acompanhar a permanência desses conceitos, conforme o período do desenvolvimento da pesquisa de doutorado, realizada pelo primeiro autor e orientado pela segunda autora deste artigo.

\section{Pesquisas que compuseram o corpus}

A seguir apresentamos as informações de análise das 26 pesquisas em ordem cronológica — objetivo, questão(ões) de pesquisa e conclusão.

Angelini (2010) - Objetivo: identificar a imagem conceitual e a definição conceitual de função, e características dos três mundos da Matemática, apresentados por oito alunos da $2^{\mathrm{a}}$ série do Ensino Médio. Questões de pesquisa:

\footnotetext{
Qual imagem de conceito de função que alunos do Ensino Médio apresentam, após terem estudado este tema na $1^{\text {a }}$ série do Ensino Médio? Qual definição de conceito de função que alunos do Ensino Médio apresentam, após terem estudado este tema na $1^{\text {a }}$ série do Ensino Médio? Quais características dos três mundos da matemática surgem nas respostas de alunos ao resolver problemas relacionados ao conceito de função? (ANGELINI, 2010, p. 21)
}

Conclusão: os 8 alunos "demonstraram dificuldades em operações aritméticas que deveriam ser consideradas elementares para a faixa etária de 15 a 17 anos" (ANGELINI, 2010, p. 168), houve a recomendação de retomar 0 aprendizado de funções com abordagem de ensino 
que leve em consideração as imagens e definições conceituais, além das características dos três mundos da Matemática entendido como uma forma de potencializar a aprendizagem.

Amorim (2011) - Objetivo: desvendar a contribuição de uma proposta de ensino, baseada nas imagens conceituais, relacionadas ao conceito de limite de uma função, (re)construídas por licenciandos em Matemática, após cursarem Análise Real.

Questão de pesquisa: "Como uma proposta de ensino, baseada nas imagens conceituais, relacionadas ao conceito de limite de uma função, (re)construídas por alunos do curso de licenciatura em Matemática, após cursarem Análise Real, pode contribuir para a aprendizagem desses alunos?" (AMORIM, 2011, p. 27)

Conclusão: contribuições ao professor de Análise, em uma proposta de ensino baseada nas imagens conceituais dos licenciandos:

Entender e situar o momento e a aprendizagem de seus alunos;

Perceber a importância de identificar e (re) significar imagens conceituais equivocadas e/ou conflitantes;

Reconhecer a necessidade de (re) construir imagens conceituais coerentes e que explorem elementos intuitivos;

Trabalhar na perspectiva de se construir definições conceituais de acordo com as definições formais;

Repensar sua prática pedagógica e planejar suas ações;

Incentivar uma postura mais crítica e ativa em seus alunos e, assim desmistificar 0 "horror" à Análise (AMORIM, 2011, p. 126-128).

Andersen (2011) - Objetivo: investigar quais processos mentais intervêm e são combinados quando os estudantes desenvolvem atividades nas quais é tratada a expressão $F(x)=\int_{a}^{x} f(t) d t$ e se os tipos de atividades propostas favorecem a compreensão das ideias centrais do referido teorema. Questões de pesquisas:

Quais processos mentais intervêm e são combinados quando se insere atividades que se apoiam em figuras construídas pelo aluno tanto em folha de papel quanto pelo software Winplot ao se tratar da expressão $F(x)=\int_{a}^{x} f(t) d t$ ? Esse tipo de atividade favorece a compreensão das ideias centrais que envolvem o Teorema Fundamental do Cálculo? (ANDERSEN, 2011, p. 28)

Conclusão: em relação aos processos mentais, os resultados da pesquisa evidenciaram que os estudantes mobilizaram os processos de visualização, representação e conversões entre diferentes representações, síntese e generalização durante a realização das atividades propostas. 
Fonseca (2011) - Objetivo: discutir e avaliar a utilização integrada do software Mathlets como ferramenta nas aulas de Matemática, em turmas da $1^{\text {a }}$ série do Ensino Médio. Questões de pesquisa:

Quais seriam as implicações educacionais decorrentes da inserção dessas inovações tecnológicas no ensino da matemática? Como o professor pode agregar a utilização de recursos tecnológicos, às suas ações da prática de ensino de matemática, com vistas à melhoria da aprendizagem dessa área de conhecimento? (FONSECA, 2011, p. 134)

Conclusão: houve a indicação da integração do software Mathlets às atividades propostas, como inovações tecnológicas, no ensino de função afim, que levou os alunos à uma autonomia crescente na realização das atividades e "que é necessário que o professor desenvolva materiais consistentes, que permitam certa adaptação, a fim de garantir a eficácia da aplicação dessas atividades, permitindo que elas, sejam adaptáveis à realidade de cada turma" (FONSECA, 2011, p. 135).

Franco (2011) - Objetivo: investigar os diversos conflitos de aprendizagem apresentados por licenciandos em Matemática, diante de um primeiro curso de Álgebra Abstrata, visando compreendê-los na perspectiva das interações entre a definição matemática formal e as imagens conceituais.

Questão de pesquisa: "O que evidenciam os conflitos de aprendizagem manifestados por alunos de licenciatura em Matemática num primeiro curso de Álgebra Abstrata, à luz das interações entre definição formal e imagens conceituais?" (FRANCO, 2011, p. 16)

Conclusão: de modo geral, os 12 alunos participantes apresentaram um rendimento satisfatório nas atividades, o que aponta desenvolvimento nos níveis do pensamento matemático. Para esse autor "em situações específicas, operavam os objetos algébricos de maneira não apenas rotineira ou repetitiva" (FRANCO, 2011, p. 87).

Janzen (2011) - Objetivo: trabalhar com provas em Geometria em um ambiente dinâmico da perspectiva do papel do professor de Ensino Superior, em seu papel de formador do pensamento matemático.

Questão de pesquisa: "Como pode o professor de Ensino Superior criar um ambiente favorável para o aprender a pensar matematicamente por meio de provas em Geometria num ambiente dinâmico com suas potencialidades? (JANZEN, 2011, p. 16)

Conclusão: foi identificado que enquanto o professor fez uso das potencialidades do 
software suas intervenções foram direcionadas em dois sentidos: de cunho organizacional e de cunho matemático. Nas intervenções de cunho matemático, a autora afirmou que dificilmente apareceram separadas, formaram uma rede unindo aspectos dinâmicos do software, do conteúdo, da especificidade das construções geométricas aliados à visualização potencializada pela utilização do software GeoGebra.

Novais (2011) - Objetivo: discutir e avaliar os efeitos de uma proposta alternativa para 0 estudo das representações das soluções de equações e sistema de equações em $R^{2}$, tomando como referência a noção de imagem conceitual. Não apresentou questão de pesquisa.

Conclusão: os elementos que compuseram sua proposta para o ensino de equações trouxeram enriquecimento à imagem de conceitual dos estudantes e fortaleceram a conexão entre as unidades cognitivas já existentes e as adquiridas ao longo do desenvolvimento dos exercícios propostos por uma sequência didática.

Santos (2011) - Objetivo: elaborar, aplicar e analisar uma sequência didática para 0 ensino da função logarítmica utilizando o software GeoGebra como estratégia pedagógica. Questões de pesquisas:

\footnotetext{
Os alunos com a sequência didática proposta neste trabalho conseguem reconhecer alguns elementos fundamentais para o estudo da função logarítmica, tais como domínio, imagem e o esboço do gráfico? Em que medida? Quais as dificuldades encontradas? Quais avanços percebidos? 0 uso do software GeoGebra como estratégia didático-pedagógica no estudo da função logarítmica contribuiu ou não para a aprendizagem dos alunos? (SANTOS, 2011, p. 18)
}

Conclusão: houve uma evolução de conhecimento das duplas, as discussões entre os alunos favoreceram o levantamento de hipóteses sobre o comportamento do gráfico da função, do domínio e da imagem, e que o uso do software como uma estratégia didático-pedagógica contribuiu para a aprendizagem dos sujeitos da pesquisa.

Bertolazi (2012) - Objetivo: investigar os processos mentais do pensamento matemático avançado manifestados em registros escritos de licenciandos em Matemática em tarefas sobre sistemas de equações lineares.

Questão de pesquisa: "Que manifestações de pensamento matemático avançado são reveladas em registros escritos de estudantes de licenciatura em Matemática ao resolverem tarefas sobres sistemas de equações lineares?" (BERTOLAZI, 2012, p. 17)

Conclusão: todos apresentaram pelo menos três processos mentais diferentes de 
representação associados ao pensamento matemático avançado, dentre nove processos que foram analisados, mas "em relação aos processos essenciais envolvendo a abstração matemática, apenas 3 estudantes dentre 17 participantes manifestaram a capacidade de formalização, generalização e síntese na resolução de tarefas sobre sistemas de equações lineares" (BERTOLAZI, 2012, p. 210).

Fonseca (2012) - Objetivo: verificar se o desenvolvimento de atividades baseadas na corporificação dos conceitos, buscando a transição entre os mundos corporificados e simbólicos, com a utilização de um software de Geometria Dinâmica, favorece a compreensão da convergência de sequências e séries.

Questão de pesquisa: "Que contribuições uma proposta pedagógica baseada na corporificação de conceitos pode trazer para a compreensão do conceito de convergência de sequências e séries em uma turma de Cálculo?" (FONSECA, 2012, p. 29)

Conclusão: ficou evidenciado que em um curso de Cálculo Diferencial e Integral, não é necessário ter como objetivo o tratamento formal, característico da última fase do desenvolvimento cognitivo, podendo ser deixado para a disciplina Análise.

Kirnev (2012) - Objetivo: investigar dificuldades relacionadas às formas de demonstrações matemáticas (diretas, contra positivas, redução ao absurdo e contraexemplo) evidenciadas em registros de graduandos de Matemática em uma universidade norte paranaense. Questão de pesquisa: "Que dificuldades graduandos de Matemática explicitam no desenvolvimento de tarefas envolvendo demonstrações?" (KIRNEV, 2012, p. 13)

Conclusão: existiram evidências de dificuldades apresentadas pelos graduandos relacionadas: "à forma de demonstração; ao conteúdo; à escrita na linguagem matemática ou materna" (p. 98), e que a pesquisa foi relevante por "explicitar dificuldades em demonstrações matemáticas que podem ser comuns a inúmeros outros graduandos de cursos de Matemática" (KIRNEV, 2012, p. 99). A autora afirma que sua pesquisa contribuiu à comunidade acadêmica com o intuito de instigar a reflexão sobre os processos de ensino e de aprendizagem acerca das demonstrações matemáticas.

Poggio (2012) - Objetivo: investigar quais são as definições que alunos do Ensino Médio apresentam sobre proporcionalidade direta e proporcionalidade inversa, bem como analisar com que características trabalham questões que envolvem tais conceitos. Questões de pesquisa: 
Qual a definição de conceito que alunos do ensino médio dão da proporcionalidade direta? Qual a imagem de conceito que alunos do ensino médio têm sobre proporcionalidade direta? Qual a definição de conceito que alunos do ensino médio dão da proporcionalidade inversa? Qual a imagem de conceito que alunos do ensino médio têm sobre proporcionalidade inversa? Com que características, entre formais, simbólicas e corporificadas, esses alunos trabalham questionamentos que envolvem a proporcionalidade direta? Com que características, entre formais, simbólicas e corporificadas esses alunos trabalham questionamentos relacionados à proporcionalidade inversa? (POGGIO, 2012, p. 11)

Conclusão: os sujeitos de pesquisa não apresentaram em "suas respostas uma imagem de conceito de proporcionalidade direta e proporcionalidade inversa, rica e diversificada, com características dos três mundos da matemática" (POGGIO, 2012, p. 208).

Prado (2012) - Objetivo: investigar o efeito da inserção do uso da calculadora nas situações de aprendizagem propostas no Ensino Fundamental - Anos Finais, à luz da teoria do pensamento matemático avançado.

Questão de pesquisa: "Que situações de aprendizagem, para os quais se sugere a inserção da calculadora no caderno do professor, podem promover no aluno desenvolvimento do pensamento matemático avançado?" (PRADO, 2012, p. 28)

Conclusão: a inserção do uso da calculadora nas situações de aprendizagem analisadas era insuficiente e para que a utilização da calculadora na resolução das situações de aprendizagem pudesse contribuir para aprendizagem dos alunos seria desejável um maior número de situações de aprendizagem para que eles tivessem um contato mais frequente com o recurso.

Yokoyama (2012) - Objetivo: analisar a compreensão de quantificação de 1 a 10 elementos de conjuntos discretos de crianças e adolescentes, com síndrome de down, e elaborar atividades que podem contribuir para o desenvolvimento dessa compreensão. Não houve questão de pesquisa.

Conclusão: a "interação entre conceitos e procedimentos foi um caminho viável para atingir uma melhor compreensão do conceito de número" (YOKOYAMA, 2012, p. 222).

Almeida (2013) - Objetivo: elaborar um panorama de preposições teóricas sobre a aprendizagem do Cálculo Diferencial e Integral existentes em artigos publicados por David Tall e colaboradores; realizar sínteses de resultados e evidenciar elementos teóricos. Questões de pesquisas: 
de Tall na análise de dificuldades de aprendizagem do Cálculo Diferencial e Integral? Quais foram nesses artigos as abordagens desenvolvidas por Tall para 0 ensino dos tópicos: infinito, números reais, limite, derivada e integral? (ALMEIDA, 2013, p. 31)

Conclusão: David Tall e seus colaboradores produziram um amplo quadro teórico que possibilitou a elaboração de modelos, que descrevem as operações cognitivas dos sujeitos (imagem conceitual e definição conceitual; noção de proceito).

Campos (2014) - Objetivo: construir e implementar algoritmos para fatoração e primalidade como ferramenta didática que possibilite o desenvolvimento das capacidades de generalização e abstração, juntamente com conceitos sobre variáveis, funções e alguns temas introdutórios sobre teoria dos números. Não houve questão de pesquisa.

Conclusão: a sequência didática proposta pode potencializar a aprendizagem dos conceitos de variável e função como meios de relacionar grandezas, utilizando-se programação de algoritmos para discutir temas da teoria dos números, com o uso das linguagens Python e Scratch e que pode possibilitar o desenvolvimento da capacidade de generalizar e abstrair, sob a perspectiva do pensamento matemático avançado.

Gereti (2014) - Objetivo: descrever e discutir indícios/características dos processos do pensamento matemático avançado evidenciados na produção escrita de estudantes de Matemática da Universidade Estadual de Londrina ao resolverem questões discursivas do ENADE (Exame Nacional de Desempenho dos Estudantes).

Questão de pesquisa: "Que processos do pensamento matemático avançado são evidenciados por estudantes de Matemática ao resolverem questões discursivas do ENADE?" (GERETI, 2014, p. 18). Conclusão: que os mesmos processos evidenciados nas respostas padrão do ENADE foram mobilizados nas resoluções de alguns estudantes, com exceção do processo de visualização.

Fernandes Junior (2014) - Objetivo: verificar e analisar as modificações nas imagens conceituais de alunos de licenciatura em Matemática durante a aplicação de atividades de ensino sobre sequências de números reais, na perspectiva da disciplina Análise.

Questão de pesquisa: "Como atividades de ensino de sequências de números reais podem modificar as imagens conceituais de alunos de Análise na Reta?" (FERNANDES JUNIOR, 2014, p. 13)

Conclusão: três dos alunos "colocaram a importância da disciplina Análise na relação 
desta com os conteúdos de Cálculo" (FERNANDES JUNIOR, 2014, p. 99); dois dos alunos acreditavam que a importância da disciplina de Análise da Reta favorece a formação do professor de Matemática. $O$ autor afirma que sua pesquisa cumpriu com o objetivo de ampliar as discussões sobre o processo de ensino e de aprendizagem na disciplina Análise, propondo também uma alternativa metodológica.

Mação (2014) - Objetivo: apresentar abordagens para o ensino de derivada. Questões de pesquisas:

\begin{abstract}
Quais abordagens aparecem em livros didáticos de Cálculo usados em algumas universidades do estado de São Paulo? Quais características dos três mundos da matemática são contempladas nessas abordagens? É possível elaborar uma abordagem inicial do conceito de derivada, pela concepção geométrica, com o objetivo de inserir o sujeito na construção de seu conhecimento e com a presença de características formais, simbólicas e corporificadas desse conceito? É possível elaborar uma abordagem inicial do conceito de derivada, pela concepção taxa, com o objetivo de inserir o sujeito na construção de seu conhecimento e com a presença de características formais, simbólicas e corporificadas desse conceito? É possível elaborar uma abordagem inicial do conceito de derivada, pela concepção aproximação, com o objetivo de inserir o sujeito na construção de seu conhecimento e com a presença de características formais, simbólicas e corporificadas desse conceito? (MAÇÃO, 2014, p. 24)
\end{abstract}

Conclusão: a maneira de ensinar o conceito de derivada como uma abordagem geométrica foi a mais usada nos dois livros didáticos de Cálculo analisados, seguida pela abordagem taxa; que ambas apresentaram características dos três mundos: corporificado, simbólico e formal.

Marins (2014) - Objetivo: identificar e discutir que indícios/características de processos do pensamento matemático avançado são manifestados por estudantes do curso de Matemática da Universidade Estadual de Londrina ao lidarem com tarefas referentes ao conteúdo de transformações lineares.

Questão de pesquisa: "Que processos do pensamento matemático avançado são manifestados nos registros escritos apresentados por estudantes do curso de Matemática ao resolverem tarefas relacionadas ao conteúdo de transformações lineares?" (MARINS, 2014, p. 16)

Conclusão: seis dos treze estudantes manifestaram características dos processos mentais do pensamento matemático avançado na maioria das tarefas e que os estudantes podem manifestar características dos processos do pensamento matemático avançado em relação a esses conceitos durante a graduação. Porém, "sete estudantes não apresentaram características 
desses processos, porque não responderam as tarefas e/ou escreveram respostas subjetivas que não mostraram algum conhecimento do assunto" (MARINS, 2014, p. 163) e que o desenvolvimento do pensamento matemático avançado pode contribuir para reflexão desses estudantes, a fim de que compreendam os conceitos matemáticos em questão e seus objetivos envolvidos.

Pinto (2014) - Objetivo: compreender como e de que forma as definições matemáticas são utilizadas em discussões entre estudantes e professores durante as apresentações em um seminário, cujos estudos enfatizavam representações gráficas e algébricas das funções e suas derivadas, elaboradas por meio de um software com representação gráfica dinâmica. Não houve questão de pesquisa.

Conclusão: "que os estudantes atribuíram significados aos distintos termos e símbolos utilizados na definição conceitual de conceitos relacionados a funções e suas derivadas, se aproximando em vários graus à definição formal desses conceitos" (PINTO, 2014, p. 121). Entretanto a autora "crê que sejam necessárias pesquisas baseadas nas realizações de experimentos em sala de aula, em que o aluno tenha a oportunidade de se expressar e interagir com seus pares e professores, privilegiando as formas de pensamento matemático" (PINTO, 2014, p. 121).

Martins Junior (2015) — Objetivo: discutir as contribuições da realização de atividades exploratórias para a aprendizagem de diversos conteúdos relacionados às derivadas de funções reais de uma variável real no ensino de Cálculo I, a partir da visualização proporcionada pelo software GeoGebra. Questão de pesquisa: "Que contribuições a realização de atividades exploratórias com o uso do GeoGebra pode trazer à aprendizagem de derivadas a partir da visualização?" (MARTINS JUNIOR, 2015, p. 19).

Conclusão: os resultados obtidos apontam que a visualização proporcionada pelo software GeoGebra contribuiu para uma ressignificação de diversos conceitos e propriedades de derivadas que são requisitados na construção de gráficos de funções reais, além de destacar a importância, nos processos de ensino e de aprendizagem de Cálculo I, um equilíbrio entre os processos visuais e os processos algébricos.

Leme (2016) - Objetivo: investigar os fluxos de pensamento na formação do pensamento matemático na aprendizagem do conceito de derivada. Questões de pesquisa: "Quais são os fluxos de pensamento, embasados na teoria dos três mundos da matemática, envolvidos na aprendizagem da derivada? Como o entendimento desses fluxos pode potencializar o aprendizado 
desse conceito?" (LEME, 2016, p.108)

Conclusão: Leme (2016) afirmou que o entendimento do desenvolvimento dos fluxos de pensamento relacionados à aprendizagem da derivada contribuiu com a Educação Matemática, pois propiciou um modo diferenciado de análise até aquele momento, em questões relacionadas ao desenvolvimento do pensamento matemático do indivíduo.

Prado (2016) - Objetivo: compreender a Álgebra Linear ensinada para na licenciatura em Matemática como um saber voltado para a formação do professor de Matemática que atuará na Educação Básica.

Questão de pesquisa: "Qual Álgebra Linear é ou poderia ser ensinada na licenciatura em Matemática, visando à prática docente na educação básica?" (PRADO, 2016, p. 31). Conclusão: a Álgebra Linear, presente nos documentos institucionais investigados, se apresentou planejada, independente das disciplinas relacionadas ao ensino e à aprendizagem em Matemática. $\mathrm{O}$ autor evidenciou elementos que podem contribuir para a formação do licenciando, são eles:

Perceber que conceitos estudados pelo licenciando, não são conceitos isolados; fazer o uso de diferentes representações; explorar o conceito de definição e o papel que a matemática exerce; estabelecer relações com as outras disciplinas; explorar o critério de verdade matemática; vivenciar diversas formas de validar conjecturas; explorar questões relacionadas ao momento histórico que possibilitou a constituição da disciplina, além de abordar noções matemáticas da educação básica à luz do pensamento matemático avançado (PRADO, 2016, p. 9).

Oliveira (2016) - Objetivo: investigar os processos de ensino e aprendizagem de Integral de Riemann em disciplinas de Análise Real, oferecidas em cursos de licenciatura e bacharelado em Matemática, a partir da utilização de softwares dinâmicos. Questão de pesquisa: "Quais são as contribuições da utilização de softwares dinâmicos para a construção do conceito de integral de Riemann no ensino de Análise Real, à luz dos processos do pensamento matemático avançado e da relação entre rigor e intuição?" (OLIVEIRA, 2016, p. 21). Conclusão: os resultados da pesquisa

apontaram que a utilização do software GeoGebra contribuiu para uma possível discussão entre professores e alunos no que tange a construção e ressignificação do conceito de integral de Riemann na Análise e/ou na transição entre o Cálculo e a Análise, [...] assim como na possibilidade de discussões de conteúdos de integral de Lebesgue, e destaca algumas possiblidades de abordagem dos processos de ensino e aprendizagem do conteúdo de integral aos professores de Análise, possibilitando-os fazer um paralelo com os significados intuitivos advindos do Cálculo, a fim de serem provados na Análise (OLIVEIRA, 2016, p.7). 
Vieira (2016) - Objetivo: identificar se dificuldades no ensino de sequências numéricas, apontadas por docentes do Ensino Superior que já ministraram o assunto em diversas disciplinas e cursos, são as enfrentadas por licenciandos em Matemática, em cuja matriz curricular sequências numéricas aparecem em duas disciplinas. Questões de pesquisa:

Quais dificuldades dos estudantes na aprendizagem de sequências numéricas são apontadas por professores que ensinam este conteúdo? (p. 33)

Quais dificuldades, relacionadas às sequências numéricas e apontadas pelos docentes entrevistados, são observadas para estudantes que trabalharam com este tema no ensino superior? (p. 33)

Quais processos do pensamento matemático avançado os estudantes participantes da pesquisa mostram ter desenvolvido no estudo de sequências numéricas? (p. 387)

Os estudantes participantes da pesquisa inter-relacionam aspectos algorítmicos, intuitivos e formais na resolução de problemas sobre sequências numéricas? (VIEIRA, 2016, p. 388)

Conclusão: identificou 16 dificuldades, que foram obtidas a partir da análise das entrevistas com docentes que ministram disciplinas de Cálculo e Análise Real; que os sujeitos de pesquisa não inter-relacionaram aspectos algorítmicos, intuitivos e formais na resolução das questões propostas sobre sequências, e que eles não mostraram ter desenvolvido os processos do pensamento matemático avançado durante a realização da atividade, como a mudança entre representações, visualização, generalização e síntese. Também identificou dificuldades com aspectos lógico-formais, relativos à elaboração de justificativas e à argumentação matemática, e erros e incompreensões em assuntos como números reais e funções.

No que se segue apresentaremos as análises, no âmbito de uma metanálise, a partir das orientações da análise de conteúdo (BARDIN, 2011).

\section{Análises}

No Quadro 1, os trabalhos estão organizados cronologicamente e em dissertações e teses:

Quadro 1: Corpus de documentos selecionados

\begin{tabular}{|c|c|c|c|}
\hline Ano & Autor (es) e título do trabalho & Instituição & Tipo \\
\hline 2010 & $\begin{array}{l}\text { ANGELINI, Norberto Machado. Funções: um estudo } \\
\text { baseado nos três mundos da Matemática }\end{array}$ & UNIBAN/SP & Mestrado \\
\hline
\end{tabular}




\begin{tabular}{|c|c|c|c|}
\hline 2011 & $\begin{array}{l}\text { AMORIM, Lilian Isabel Ferreira. A (re)construção do conceito } \\
\text { de Limite do Cálculo para Análise: um estudo com alunos do } \\
\text { curso de licenciatura em Matemática }\end{array}$ & UFOP/MG & Mestrado \\
\hline 2011 & $\begin{array}{l}\text { ANDERSEN, Érika. As ideias centrais do Teorema } \\
\text { Fundamental do Cálculo mobilizadas por alunos de } \\
\text { licenciatura em Matemática }\end{array}$ & PUC/SP & Mestrado \\
\hline 2011 & $\begin{array}{l}\text { FONSECA, Vilmar Gomes da. O uso de tecnologias no } \\
\text { Ensino Médio: a integração de Mathlets no ensino de função } \\
\text { afim }\end{array}$ & UFRJ/RJ & Mestrado \\
\hline 2011 & $\begin{array}{l}\text { FRANCO, Hernando José Rocha. Os diversos conflitos } \\
\text { observados em alguns alunos de licenciatura num curso de } \\
\text { Álgebra: identificação e análise }\end{array}$ & UFJF/MG & Mestrado \\
\hline 2011 & $\begin{array}{l}\text { NOVAIS, André Seixas de. Equações indeterminadas e } \\
\text { Lugares Geométricos: uma proposta alternativa para } 0 \\
\text { estudo de equações em } R^{2}\end{array}$ & UFRJ/RJ & Mestrado \\
\hline 2011 & $\begin{array}{l}\text { SANTOS, Adriana Tiago Castro dos. O ensino da função } \\
\text { logarítmica por meio de uma sequência didática ao explorar } \\
\text { suas representações com o uso do software GeoGebra }\end{array}$ & PUC/SP & Mestrado \\
\hline 2012 & $\begin{array}{l}\text { BERTOLAZI, Kátia Socorro. Conhecimentos e } \\
\text { compreensões revelados por estudantes de licenciatura em } \\
\text { Matemática sobre sistemas de equações lineares }\end{array}$ & UEL/PR & Mestrado \\
\hline 2012 & $\begin{array}{l}\text { FONSECA, Daila Silva Seabra de Moura. Convergências de } \\
\text { sequências e séries numéricas no Cálculo: um trabalho } \\
\text { visando a corporificação dos conceitos }\end{array}$ & UFOP/MG & Mestrado \\
\hline 2012 & $\begin{array}{l}\text { KIRNEV, Debora Cristiane Barbosa. } \\
\text { evidenciadas em registros escritos a respeito de } \\
\text { demonstrações matemáticas }\end{array}$ & UEL/PR & Mestrado \\
\hline 2012 & $\begin{array}{l}\text { POGGIO, Ana Maria Pereira Pinto. Um diagnóstico sobre o } \\
\text { conceito de proporcionalidade de alunos do Ensino Médio na } \\
\text { perspectiva dos três mundos da Matemática }\end{array}$ & UNIBAN/SP & Mestrado \\
\hline 2012 & $\begin{array}{l}\text { PRADO, Sonia de Cassia Santos. O uso da calculadora e o } \\
\text { pensamento matemático avançado: uma análise a partir das } \\
\text { situações de aprendizagem nos Cadernos do Professor de } \\
\text { Matemática }\end{array}$ & PUC/SP & Mestrado \\
\hline 2013 & $\begin{array}{l}\text { ALMEIDA, Marcio Vieira de. Um panorama de artigos sobre } \\
\text { a aprendizagem do Cálculo Diferencial e Integral na } \\
\text { perspectiva de David Tall }\end{array}$ & PUC/SP & Mestrado \\
\hline 2014 & $\begin{array}{l}\text { CAMPOS, Jean Peixoto. Algoritmos para fatoração e } \\
\text { primalidade como ferramenta didática para o ensino de } \\
\text { Matemática }\end{array}$ & UNIR/RO & Mestrado \\
\hline
\end{tabular}




\begin{tabular}{|c|c|c|c|}
\hline 2014 & $\begin{array}{l}\text { GERETI, Laís Cristina Viel. Processos do pensamento } \\
\text { matemático avançado evidenciados em resoluções de } \\
\text { questões do ENADE }\end{array}$ & UEL/PR & Mestrado \\
\hline 2014 & $\begin{array}{l}\text { FERNANDES JUNIOR, Valter Costa. Repensando o ensino } \\
\text { de Análise: reações, impressões e modificações nas } \\
\text { imagens de conceito de alunos frente a atividades de ensino } \\
\text { sobre sequências de números reais }\end{array}$ & UFJF/MG & Mestrado \\
\hline 2014 & $\begin{array}{l}\text { MAÇÃO, Douglas Paes. Uma proposta de ensino para } 0 \\
\text { conceito de Derivada }\end{array}$ & ANHANGUERA/SP & Mestrado \\
\hline 2014 & $\begin{array}{l}\text { MARINS, Alessandra Senes. Pensamento matemático } \\
\text { avançado em tarefas envolvendo transformações lineares }\end{array}$ & UEL/PR & Mestrado \\
\hline 2014 & $\begin{array}{l}\text { PINTO, Rieuse Lopes. Definições matemáticas sobre } \\
\text { funções e suas derivadas como um eixo de discussão para } \\
\text { o ensino e a aprendizagem do Cálculo }\end{array}$ & UFOP/MG & Mestrado \\
\hline 2015 & $\begin{array}{l}\text { MARTINS JUNIOR, José Cirqueira. Ensino de derivadas em } \\
\text { Cálculo I: aprendizagem a partir da visualização com o uso } \\
\text { do GeoGebra }\end{array}$ & UFOP/MG & Mestrado \\
\hline 2016 & $\begin{array}{l}\text { OLIVEIRA, João Lucas de. A utilização de softwares } \\
\text { dinâmicos no ensino de Análise Real: um estudo sobre a } \\
\text { construção do conceito de Integral de Riemann }\end{array}$ & UFOP/MG & Mestrado \\
\hline 2011 & $\begin{array}{l}\text { JANZEN, Elen Andrea. O papel do professor na formação do } \\
\text { pensamento matemático de estudantes durante a } \\
\text { construção de provas em um ambiente de Geometria } \\
\text { Dinâmica }\end{array}$ & UFPR/PR & Doutorado \\
\hline 2012 & $\begin{array}{l}\text { YOKOYAMA, Leo Akio. Uma abordagem multissensorial } \\
\text { para o desenvolvimento do conceito de número natural em } \\
\text { indivíduos com Síndrome de Down }\end{array}$ & UNIBAN/SP & Doutorado \\
\hline 2016 & $\begin{array}{l}\text { LEME, Jayme do Carmo Macedo. Aprendizagem da } \\
\text { Derivada: uma perspectiva de análise pelos fluxos de } \\
\text { pensamento }\end{array}$ & PUC/SP & Doutorado \\
\hline 2016 & $\begin{array}{l}\text { PRADO, Eneias de Almeida. Álgebra Linear na licenciatura } \\
\text { em Matemática: contribuições para formação do profissional } \\
\text { da Educação Básica }\end{array}$ & PUC/SP & Doutorado \\
\hline 2016 & $\begin{array}{l}\text { VIEIRA, Willian. Do Cálculo à Análise Real: um diagnóstico } \\
\text { dos processos de ensino e de aprendizagem de sequências } \\
\text { numéricas }\end{array}$ & ANHANGUERA/SP & Doutorado \\
\hline
\end{tabular}

Fonte: Adaptado de Carmo (2018, p. 45-47)

O Quadro 1 indica que as pesquisas se concentraram na região sudeste do Brasil, totalizando 20 trabalhos (aproximadamente $77 \%$ ), sendo a maioria do tipo dissertação. No Quadro 
2 o foco está no tema/conteúdo matemático pesquisado e no nível de ensino dessas produções.

Quadro 2: Algumas características das pesquisas selecionadas

\begin{tabular}{|c|c|c|c|}
\hline Ano & Autor(a) & Tema / conteúdo & Nível \\
\hline 2010 & Angelini & Conceito de função & Ensino Médio \\
\hline 2011 & Amorim & Limites de funções reais de uma variável & Ensino Superior \\
\hline 2011 & Andersen & Teorema Fundamental do Cálculo & Ensino Superior \\
\hline 2011 & Fonseca & Função afim & Ensino Médio \\
\hline 2011 & Franco & Álgebra Abstrata & Ensino Superior \\
\hline 2011 & Novais & Equações indeterminadas e lugares geométricos & Ensino Superior \\
\hline 2011 & Santos & $\begin{array}{c}\text { Sequência didática para ensinar função } \\
\text { logarítmica }\end{array}$ & Ensino Médio \\
\hline 2012 & Bertolazi & Sistemas de equações lineares & Ensino Superior \\
\hline 2012 & Fonseca & $\begin{array}{l}\text { Convergência de sequências e séries numéricas } \\
\text { infinitas }\end{array}$ & Ensino Superior \\
\hline 2012 & Kirnev & Demonstrações matemáticas & Ensino Superior \\
\hline 2012 & Poggio & Conceito de proporcionalidade direta e inversa & Ensino Médio \\
\hline 2012 & Prado & Uso da calculadora & $\begin{array}{c}\text { Ensino } \\
\text { Fundamental }\end{array}$ \\
\hline 2013 & Almeida & Cálculo Diferencial e Integral & Ensino Superior \\
\hline 2014 & Campos & Funções e teoria dos números & Ensino Médio \\
\hline 2014 & Gereti & Questões discursivas do ENADE & Ensino Superior \\
\hline 2014 & Fernandes Junior & Ensino de Análise Matemática & Ensino Superior \\
\hline 2014 & Mação & Ensino de derivada & Ensino Superior \\
\hline 2014 & Marins & Transformações lineares & Ensino Superior \\
\hline 2014 & Pinto & Funções e suas derivadas & Ensino Superior \\
\hline 2015 & Martins Junior & Ensino e aprendizagem de derivada & Ensino Superior \\
\hline 2016 & Oliveira & Integral de Riemann & Ensino Superior \\
\hline 2011 & Janzen & Construções de provas geométricas & Ensino Superior \\
\hline
\end{tabular}




\begin{tabular}{|l|c|c|c|}
\hline 2012 & Yokoyama & Ensino do conceito de número natural & $\begin{array}{c}\text { Ensino } \\
\text { Fundamental }\end{array}$ \\
\hline 2016 & Leme & Aprendizagem do conceito de derivada & Ensino Superior \\
\hline 2016 & Prado & $\begin{array}{c}\text { Disciplina de Álgebra Linear na licenciatura em } \\
\text { Matemática }\end{array}$ & Ensino Superior \\
\hline 2016 & Vieira & Sequências numéricas & Ensino Superior \\
\hline
\end{tabular}

Fonte: Adaptado de Carmo (2018, p. 48)

As informações do Quadro 2 possibilitam afirmarmos que as dissertações e teses se concentraram em discussões com abordagem para o Ensino Superior (19 no Ensino Superior, 5 no Ensino Médio, e 2 no Ensino Fundamental) totalizando, aproximadamente, $73 \%$ das pesquisas analisadas. Esse porcentual é um indício de que esses pesquisadores consideram que as características do pensamento matemático avançado se revelam no Ensino Superior, conforme Tall (2002).

Observamos que a maioria dos trabalhos do Ensino Superior se concentra em conteúdos de Análise Matemática (11 trabalhos), de Álgebra (5 trabalhos), em provas e demonstrações (2 trabalhos), e em vários conteúdos de questões discursivas do ENADE (1 trabalho). Algumas dessas pesquisas utilizaram vários conceitos matemáticos para estudar as dificuldades de ensino e de aprendizagem no nível superior ou para sugerir abordagens para o ensino desses conceitos/conteúdos.

No Quadro 3 indicamos o tipo de investigação (teórica ou empírica), os sujeitos de pesquisa e/ou documentos e os principais referencial(is) teórico(s) utilizado(s).

Quadro 3: Algumas características das pesquisas selecionadas

\begin{tabular}{|c|c|c|c|}
\hline Ano & Autor (a) & Sujeitos / Documentos & $\begin{array}{l}\text { Referencial(is) } \\
\text { teórico(s) }\end{array}$ \\
\hline 2010 & Angelini & 8 estudantes da $2^{\mathrm{a}}$ série do Ensino Médio & $\begin{array}{l}\text { David Tall; Shlomo } \\
\text { Vinner }\end{array}$ \\
\hline 2011 & Amorim & $\begin{array}{c}\text { Análise de livros de Cálculo Diferencial e Integral e } \\
9 \text { estudantes de licenciatura em Matemática }\end{array}$ & $\begin{array}{l}\text { David Tall; Shlomo } \\
\text { Vinner }\end{array}$ \\
\hline 2011 & Andersen & 14 estudantes de licenciatura em Matemática & Tommy Dreyfus \\
\hline 2011 & Fonseca & Estudantes da $1^{\mathrm{a}}$ série do Ensino Médio & $\begin{array}{l}\text { David Tall; Shlomo } \\
\text { Vinner; Raymond Duval }\end{array}$ \\
\hline
\end{tabular}




\begin{tabular}{|c|c|c|c|}
\hline 2011 & Franco & 12 estudantes de licenciatura em Matemática & $\begin{array}{l}\text { Tommy Dreyfus; David } \\
\text { Tall; Shlomo Vinner }\end{array}$ \\
\hline 2011 & Novais & Estudantes do curso de Matemática & $\begin{array}{l}\text { David Tall; Shlomo } \\
\text { Vinner }\end{array}$ \\
\hline 2011 & Santos & 6 estudantes de uma $3^{\mathrm{a}}$ série do Ensino Médio & $\begin{array}{l}\text { Tommy Dreyfus; } \\
\text { Raymond Duval }\end{array}$ \\
\hline 2012 & Bertolazi & 17 estudantes de licenciatura em Matemática & $\begin{array}{l}\text { Tommy Dreyfus; Lauren } \\
\text { Resnick; Paulo Freire }\end{array}$ \\
\hline 2012 & Fonseca & $\begin{array}{c}\text { Estudantes da disciplina de Cálculo II do curso de } \\
\text { Engenharia de Produção }\end{array}$ & $\begin{array}{l}\text { Tommy Dreyfus; David } \\
\text { Tall }\end{array}$ \\
\hline 2012 & Kirnev & 13 estudantes de licenciatura em Matemática & $\begin{array}{l}\text { Nicolas Balacheff; } \\
\text { Tommy Dreyfus }\end{array}$ \\
\hline 2012 & Poggio & $\begin{array}{c}51 \text { estudantes de } 2 \text { turmas da } 3^{\mathrm{a}} \text { série do Ensino } \\
\text { Médio }\end{array}$ & $\begin{array}{l}\text { David Tall; Shlomo } \\
\text { Vinner }\end{array}$ \\
\hline 2012 & Prado & $\begin{array}{l}\text { Caderno do Professor do Ensino Fundamental - } \\
\text { Anos Finais da SEE/SP }\end{array}$ & Tommy Dreyfus \\
\hline 2013 & Almeida & Artigos de David Tall e colaboradores & $\begin{array}{l}\text { David Tall e } \\
\text { colaboradores }\end{array}$ \\
\hline 2014 & Campos & Sequência didática & $\begin{array}{c}\text { Seymour Papert; Tommy } \\
\text { Dreyfus }\end{array}$ \\
\hline 2014 & Gereti & 13 estudantes de licenciatura em Matemática & Tommy Dreyfus \\
\hline 2014 & $\begin{array}{l}\text { Fernandes } \\
\text { Junior }\end{array}$ & 6 estudantes de licenciatura em Matemática & $\begin{array}{l}\text { David Tall; Shlomo } \\
\text { Vinner }\end{array}$ \\
\hline 2014 & Mação & $\begin{array}{c}\text { Análise de } 2 \text { livros de Cálculo Diferencial e Integral } \\
\text { e proposta de ensino }\end{array}$ & David Tall \\
\hline 2014 & Marins & 13 estudantes do bacharelado em Matemática & $\begin{array}{l}\text { Tommy Dreyfus; David } \\
\text { Tall }\end{array}$ \\
\hline 2014 & Pinto & 38 estudantes do curso de Sistema de Informação & $\begin{array}{c}\text { David Tall; Shlomo } \\
\text { Vinner }\end{array}$ \\
\hline 2015 & $\begin{array}{l}\text { Martins } \\
\text { Junior }\end{array}$ & 4 professores de Matemática do Ensino Superior & $\begin{array}{l}\text { Tommy Dreyfus; David } \\
\text { Tall }\end{array}$ \\
\hline 2016 & Oliveira & $\begin{array}{l}\text { Estudantes de licenciatura e bacharelado em } \\
\text { Matemática }\end{array}$ & $\begin{array}{l}\text { Tommy Dreyfus; David } \\
\text { Tall; Frederico Reis }\end{array}$ \\
\hline 2011 & Janzen & 2 professores universitários da Alemanha & Tommy Dreyfus \\
\hline
\end{tabular}




\begin{tabular}{|c|c|c|c|}
\hline 2012 & Yokoyama & 8 crianças/adolescentes com síndrome de down & $\begin{array}{c}\text { David Tall; Shlomo } \\
\text { Vinner }\end{array}$ \\
\hline 2016 & Leme & Produção de materiais & David Tall \\
\hline 2016 & Prado & $\begin{array}{c}\text { Documentos de } 6 \text { universidades e entrevista com } \\
8 \text { professores do curso de licenciatura Matemática }\end{array}$ & Tommy Dreyfus \\
\hline 2016 & Vieira & $\begin{array}{r}\text { Análise de livros, entrevistas com docentes e, } \\
\text { questionários e entrevistas com licenciandos em } \\
\text { Matemática }\end{array}$ & $\begin{array}{c}\text { Tommy Dreyfus; Efraim } \\
\text { Fischbein }\end{array}$ \\
\hline
\end{tabular}

Fonte: Carmo (2018, p. 49-51)

Constatamos que a maioria das pesquisas se constituiu de investigações empíricas totalizando 21 trabalhos (aproximadamente 81\%). Entre esses trabalhos, 4 tinham professores como sujeito de pesquisa, e 13 trabalhos os sujeitos eram alunos de graduação (1 pesquisa entrevistou docentes e aplicou questionários e entrevistas aos licenciandos), 4 trabalhos tomaram alunos de Ensino Médio e 1 trabalho alunos do Ensino Fundamental. Vale ressaltar que algumas dessas pesquisas foram documentais e aplicadas e 5 pesquisas foram apenas documentais.

Das 13 investigações cujos sujeitos eram alunos de graduação, 11 foram com estudantes de Matemática, 1 com estudantes de Engenharia e 1 com estudantes de Sistema de Informação. Constatamos uma concentração de pesquisas com estudantes de Matemática, aproximadamente $85 \%$ das pesquisas com alunos de graduação.

Podemos notar que as pesquisas, selecionadas no corpus deste artigo, buscaram caminhos para garantir a aprendizagem dos estudantes ou para orientar os professores em melhores estratégias para o desenvolvimento do pensamento matemático avançado em conteúdos matemáticos que necessitam deste tipo de pensamento, algumas pesquisas utilizaram esse referencial teórico de modo inadequado, prejudicando seus resultados. No entanto, nessas pesquisas não são apresentadas justificativas para a importância do desenvolvimento do PMA em determinado nível de ensino, por exemplo na licenciatura em Matemática - o que isso irá influenciar na prática do futuro professor, esse seria um tema para ser estudado em futuras pesquisas.

\section{Considerações Finais}

As análises permitem afirmarmos que as pesquisas brasileiras selecionadas neste estudo 
se concentraram na Região Sudeste, indicando maior interferência dessa teoria nessa região. Valeria ampliar a pesquisa para buscar mais informações do porquê isso ocorreu. As pesquisas se concentraram no Ensino Superior, podendo indicar que os pesquisadores investigados parecem associar o desenvolvimento do pensamento matemático avançado a este nível de ensino, de acordo com Tall (2002), e que esse tipo de desenvolvimento de pensamento provoca dificuldades de ensino e de aprendizagem nas disciplinas relacionadas ao Cálculo e a Álgebra. Com relação ao tipo de pesquisa, teórica ou empírica, podemos afirmar que há a necessidade de pesquisas teóricas relacionadas ao assunto, visto que a maioria das pesquisas selecionadas foi do tipo empírica (aplicada).

Podemos afirmar que a teoria do pensamento matemático avançado (PMA) e seus complementos apresenta constructos teóricos para compreender o processo de formação do pensamento matemático, e é base para potencializar estratégias de ensino que promovam de forma eficiente a aprendizagem dos conceitos matemáticos. A diversidade de concepções acomoda o estudo cognitivo e concepções teóricas dos pesquisadores brasileiros. Para nós, os elementos do PMA indicam complexidade conceitual, em geral encontrados nos cursos superiores.

\section{Referências}

ALMEIDA, Marcio Vieira de. Um panorama de artigos sobre a aprendizagem do Cálculo Diferencial e Integral na perspectiva de David Tall. 2013. 154f. Dissertação (Mestrado em Educação Matemática) - Faculdade de Ciências Exatas e Tecnologias. Pontifícia Universidade Católica de São Paulo. São Paulo.

AMORIM, Lilian Isabel Ferreira. A (re)construção do conceito de limite do cálculo para análise: um estudo com alunos do curso de licenciatura em Matemática. 2011. 134f. Dissertação (Mestrado em Educação Matemática) — Instituto de Ciências Exatas e Biológicas. Universidade Federal de Ouro Preto. Ouro Preto.

ANDERSEN, Érika. As ideias centrais do Teorema Fundamental do Cálculo mobilizadas por alunos de licenciatura em Matemática. 2011.128f. Dissertação (Mestrado em Educação Matemática) - Faculdade de Ciências Exatas e Tecnologias. Pontifícia Universidade Católica de São Paulo. São Paulo.

ANGELINI, Norberto Machado. Funções: um estudo baseado nos três mundos da Matemática. 2010. 219f. Dissertação (Mestrado em Educação Matemática) — Universidade Bandeirante de São Paulo. São Paulo.

BARDIN, Laurence. Análise de conteúdo. Tradução de Luís Antero Reto, Augusto Pinheiro. 2. reimp. São Paulo: Edições 70, 2011. 
BERTOLAZI, Kátia Socorro. Conhecimentos e compreensões revelados por estudantes de licenciatura em Matemática sobre sistemas de equações lineares. 2012. 229f. Dissertação (Mestrado em Ensino de Ciências e Educação Matemática) - Centro de Ciências Exatas. Universidade Estadual de Londrina. Londrina.

CAMPOS, Jean Peixoto. Algoritmos para fatoração e primalidade como ferramenta didática para o ensino de Matemática. 2014. 95f. Dissertação (Mestrado Profissional em Matemática em Rede Nacional) - Núcleo de Ciências Exatas e da Terra. Universidade Federal de Rondônia. Porto Velho.

CARMO, Paulo Ferreira do. Pensamento matemático avançado: como essa noção repercute em dissertações e teses brasileiras? 2018. 128f. Tese (Doutorado em Educação Matemática) Faculdade de Ciências Exatas e Tecnologias. Pontifícia Universidade Católica de São Paulo. São Paulo

DREYFUS, Tommy. Advanced Mathematical Thinking Process. In TALL, David Orme. (Ed). Advanced Mathematical Thinking. Dordrecht: Kluwer, 2002, p. 25-41.

FERNANDES JUNIOR, Valter Costa. Repensando o ensino de Análise: reações, impressões e modificações nas imagens de conceito de alunos frente a atividades de ensino sobre sequências de números reais. 2014. 111f. Dissertação (Mestrado em Educação Matemática) - Centro de Ciências Exatas.Universidade Federal de Juiz de Fora. Juiz de Fora.

FIORENTINI, Dario; LORENZATO, Sergio. Investigação em Educação Matemática: percursos teóricos e metodológicos. 3 ed. rev. Campinas: Autores Associados, 2012.

FONSECA, Daila Silva Seabra de Moura. Convergências de sequências e séries numéricas no Cálculo: um trabalho visando a corporificação dos conceitos. 2012. 210f. Dissertação (Mestrado em Educação Matemática) - Instituto de Ciências Exatas e Biológicas. Universidade Federal de Ouro Preto. Ouro Preto.

FONSECA, Vilmar Gomes da. O uso de tecnologias no Ensino Médio: a integração de Mathlets no ensino de função afim. 2011. 152f. Dissertação (Mestrado em Ensino de Matemática) — Instituto de Matemática. Universidade Federal do Rio de Janeiro. Rio de Janeiro.

FRANCO, Hernando José Rocha. Os diversos conflitos observados em alguns alunos de licenciatura num curso de Álgebra: identificação e análise. 2011. 101f. Dissertação (Mestrado em Educação Matemática) - Instituto de Ciências Exatas. Universidade Federal de Juiz de Fora. Juiz de Fora.

GERETI, Laís Cristina Viel. Processos do pensamento matemático avançado evidenciados em resoluções de questões do ENADE. 2014. 139f. Dissertação (Mestrado em Ensino de Ciências e Educação Matemática) - Centro de Ciências Exatas. Universidade Estadual de Londrina. Londrina.

GRAY, Eddie Martin; TALL, David Orme. Duality, ambiguity and flexibility: a "proceptual" view of simple arithmetic. The Journal for Research in Mathematics Education, v. 26, n. 2, p. 115-141, mar. 1994.

JANZEN, Elen Andrea. O papel do professor na formação do pensamento matemático de 
estudantes durante a construção de provas em um ambiente de Geometria Dinâmica. 2011. 194f. Tese (Doutorado em Educação) — Setor de Educação. Universidade Federal do Paraná. Curitiba.

KIRNEV, Debora Cristiane Barbosa. Dificuldades evidenciadas em registros escritos a respeito de demonstrações matemáticas. 2012. 113f. Dissertação (Mestrado em Ensino de Ciências e Educação Matemática) - Centro de Ciências Exatas. Universidade Estadual de Londrina. Londrina.

LEME, Jayme do Carmo Macedo. Aprendizagem da Derivada: uma perspectiva de análise pelos fluxos de pensamento. 2016. 117f. Tese (Doutorado em Educação Matemática) — Faculdade de Ciências Exatas e Tecnologias. Pontifícia Universidade Católica de São Paulo. São Paulo.

MAÇÃO, Douglas Paes. Uma proposta de ensino para o conceito de Derivada. 2014. $166 f$. Dissertação (Mestrado em Educação Matemática) — Universidade Anhanguera de São Paulo. São Paulo.

MARINS, Alessandra Senes. Pensamento matemático avançado em tarefas envolvendo transformações lineares. 2014. 171f. Dissertação (Mestrado em Ensino de Ciências e Educação Matemática) - Centro de Ciências Exatas. Universidade Estadual de Londrina. Londrina.

MARTINS JUNIOR, José Cirqueira. Ensino de derivadas em Cálculo l: aprendizagem a partir da visualização com o uso do GeoGebra. 2015. 123f. Dissertação (Mestrado em Educação Matemática) - Instituto de Ciências Exatas e Biológicas. Universidade Federal de Ouro Preto. Ouro Preto.

MEYER, Cristina. Derivada/reta tangente: imagem conceitual e definição conceitual. 2003. $159 f$. Dissertação (Mestrado em Educação Matemática) - Faculdade de Ciências Exatas e Tecnologias. Pontifícia Universidade Católica de São Paulo. São Paulo.

NOVAIS, André Seixas de. Equações indeterminadas e lugares geométricos: uma proposta alternativa para o estudo de equações em $R^{2}$. 2011. 236f. Dissertação (Mestrado em Ensino de Matemática) - Instituto de Matemática. Universidade Federal do Rio de Janeiro. Rio de Janeiro.

OLIVEIRA, João Lucas de. A utilização de softwares dinâmicos no ensino de análise real: um estudo sobre a construção do conceito de Integral de Riemann. 2016. 143f. Dissertação (Mestrado em Educação Matemática) - Instituto de Ciências Exatas e Biológicas. Universidade Federal de Ouro Preto. Ouro Preto.

PINTO, Rieuse Lopes. Definições matemáticas sobre funções e suas derivadas como um eixo de discussão para o ensino e a aprendizagem do Cálculo. 2014. 144f. Dissertação (Mestrado Profissional em Educação Matemática) — Instituto de Ciências Exatas e Biológicas. Universidade Federal de Ouro Preto. Ouro Preto.

POGGIO, Ana Maria Pereira Pinto. Um diagnóstico sobre o conceito de Proporcionalidade de alunos do Ensino Médio na perspectiva dos três mundos da Matemática. 2012. 237f. Dissertação (Mestrado em Educação Matemática) — Universidade Bandeirante de São Paulo. São Paulo.

PRADO, Eneias de Almeida. Álgebra Linear na licenciatura em Matemática: contribuições para formação do profissional da Educação Básica. 2016. 254f. Tese (Doutorado em Educação Matemática) - Faculdade de Ciências Exatas e Tecnologias. Pontifícia Universidade Católica de 
São Paulo. São Paulo.

PRADO, Sonia de Cassia Santos. O uso da calculadora e o pensamento matemático avançado: uma análise a partir das situações de aprendizagem nos Cadernos do Professor de Matemática. 2012. 186f. Dissertação (Mestrado em Educação Matemática) - Faculdade de Ciências Exatas e Tecnologias. Pontifícia Universidade Católica de São Paulo. São Paulo.

SANTOS, Adriana Tiago Castro dos. O ensino da função logarítmica por meio de uma sequência didática ao explorar suas representações com o uso do software GeoGebra. 2011. 200f. Dissertação (Mestrado em Educação Matemática) - Faculdade de Ciências Exatas e Tecnologias. Pontifícia Universidade Católica de São Paulo. São Paulo.

TALL, David Orme. Building theories: the three worlds of Mathematics. For the Learning of Mathematics, New Westminster, v. 24, n. 1, p. 29-32, mar. 2004.

TALL, David Orme. The psychology of advanced mathematical thinking. In TALL, David Orme. (Ed). Advanced Mathematical Thinking. Dordrecht: Kluwer, 2002. p. 3-20.

TALL, David Orme; VINNER, Shlomo. Concept image and concept definition in Mathematics, with special reference to limits and continuity. Educational Studies in Mathematics, n. 12, p. 151-169, mayo 1981.

VIEIRA, Willian. Do Cálculo à Análise Real: um diagnóstico dos processos de ensino e de aprendizagem de sequências numéricas. 2016. 452f. Tese (Doutorado em Educação Matemática) - Universidade Anhanguera. São Paulo.

YOKOYAMA, Leo Akio. Uma abordagem multissensorial para o desenvolvimento do conceito de número natural em indivíduos com síndrome de down. 2012. 230f. Tese (Doutorado em Educação Matemática) — Universidade Bandeirante de São Paulo. São Paulo. 